\title{
Protecting elite athletes in extreme and challenging environments: advancing the dialogue
}

\author{
Michael F Bergeron, ${ }^{1,2}$ Lars Engebretsen $^{3}$
}

\begin{abstract}
Watching the Olympic athlete powering through the water or down a mountain, sprinting on the track with unimaginable speed and efficiency or demonstrating any number of other seemingly super-human feats, it is hard for some to imagine the challenges these elite athletes face that threaten their performance and health and safety. Even at the Olympic or world championship level, environmental conditions can readily erase years of training and preparation when these circumstances beyond the control of the athlete are unexpectedly more than the athlete is accustomed to and can tolerate.
\end{abstract}

The International Olympic Committee (IOC) Medical Commission recognises this, as do the International Sport Federations. New guidelines to minimise clinical risk and injury surveillance tracking systems have been developed and incorporated. ${ }^{1}$ But more needs to be done, as evidenced by periodic reports of significant performance limitations and sometimes severe or, albeit rare, near-catastrophic incidents of environment-related illness or injury. Notably, the death of 26-year-old Fran Crippen during an open-water race in Abu Dhabi in 2010 speaks of how extreme conditions can challenge an elite athlete to the point of an eventual devastating outcome. Other swimmers at that event complained of very high air and water temperatures and reportedly experienced significant dehydration, excessive fatigue and disorientation, and some were taken to the hospital.

In response to these and other environmental challenges, an IOC consensus meeting took place at the IOC headquarters

\footnotetext{
${ }^{1}$ National Youth Sports Health \& Safety Institute, USA ${ }^{2}$ Department of Paediatrics, Sanford School of Medicine of The University of South Dakota, Sanford Children's Health Research Center, Sioux Falls, SD, USA

${ }^{3}$ Department of Orthopeadic Surgery, University of Oslo/ IOC Medical \& Scientific Department, Oslo, Norway
}

Correspondence to Professor Lars Engebretsen, Department of Orthopeadic Surgery, University of Oslo/ IOC Medical \& Scientific Department, Kirkeveien 111,

Oslo 0407, Norway; lars.engebretsen@medisin.uio.no in Lausanne, Switzerland from 30 November to 2 December 2011. From that meeting, a consensus statement on thermoregulatory (in the heat and cold) and altitude challenges for the high-level athlete was developed (see page 770 ). ${ }^{2}$ To the critical issues raised by the Crippen death, hyperthermic-related challenges facing open-water swimmers and Fédération Internationale de Natation current open-water swimming guidelines are detailed in the consensus statement and further in this special issue by Mountjoy et al (see page 800). ${ }^{3}$ Other sports such as athletics, football, tennis, volleyball and triathlon are also specifically featured in this special issue, as well as the remaining challenging questions that need to be addressed with comprehensive new sport-specific research.

Along with a highlight of the benefits of heat acclimatisation and the predictive value of individual responses, there is also an emphasis on the need for new guidelines for managing heat stress in elite sports. At the other temperature extreme, two articles focus on sports training and competition in the cold, where respiratory problems can be particularly prominent and competing athletes can incur significant performance decrements and hypothermia during open-water swimming or frostbite (albeit, the risk is usually small) in some winter sports. Altitude can present another unique set of challenges for those athletes who are exposed during competition or with intermittent training at higher elevations to gain purported performance advantages. However, as noted here, events are not held at altitudes where acute mountain sickness is typically severe or other serious clinical problems arise. Lastly, new research is presented on a common hypoxic exposure strategy and consequent changes in haemoglobin mass and their effects on aerobic power.

While the featured consensus paper and other contributions included here are not comprehensively inclusive of all environment-related challenges facing Olympic and other world-class athletes, highlighting these selected barriers and associated clinical risks keeps active a critical dialogue that serves to better protect elite athletes and provides the best opportunity for them to perform optimally. We welcome additional input and contributions from others who are examining high-level athletes in practical yet extreme environmental scenarios, so that we can better understand athlete responses and strain and improve evidence-based guidelines to reduce clinical problems and injury risk even further. To all readers, we hope you find this special IOC-supported Injury Prevention and Health Protection issue of BJSM interesting and useful in educating athletes, coaches and event organisers, as well as in your research and clinical practice. To this end, we genuinely and enthusiastically applaud your commitment to sports and athlete safety.

\section{Competing interests None}

Provenance and peer review Commissioned; internally peer reviewed.

Accepted 21 June 2012

Br J Sports Med 2012;46:769.

doi:10.1136/bjsports-2012-091457

\section{REFERENCES}

1. Steffen K, Soligard T, Engebretsen L. Health protection of the Olympic athlete. $\mathrm{Br} J$ Sports Med 2012;46:466-70.

2. Bergeron MF, Bahr R, Bärtsch $P$, et al. International Olympic Committee consensus statement on thermoregulatory and altitude challenges for the high-level athlete. Br J Sports Med 2012;46:770-779.

3. Mountjoy M, Alonso J-M, Bergeron MF, et al. Hyperthermic-related challenges in aquatics, athletics, football, tennis and triathlon. $\mathrm{Br} J$ Sports Med 2012;46:800-804. 